\title{
The QCD running coupling at all scales and the connection between hadron masses and $\Lambda_{s}$
}

\section{A. Deur}

Received: date / Accepted: date

\begin{abstract}
We report on recent experimental and theoretical developments in our understanding of the QCD running coupling $\alpha_{s}$ in QCD's nonperturbative regime. They allow us to analytically compute the hadron mass spectrum, with $\Lambda_{s}$ the only input necessary to this determination. The computed spectra agrees well with experimental data.
\end{abstract}

Keywords nonperturbative QCD · strong coupling · hadron spectrum · AdS/CFT

\section{Introduction}

The strong coupling $\alpha_{s}$ sets the magnitude of the strong interaction and triggers the onsets of asymptotic freedom and confinement. As such, $\alpha_{s}$ is a central quantity to quantum chromodynamics (QCD), the gauge theory of the strong interaction. However, while $\alpha_{s}$ is well understood at high energy where its smallness $\left(\alpha_{s}<0.2\right.$ at momentum transfers $\left.Q>10 \mathrm{GeV}\right)$ allows us to employ perturbative technics, it is much less understood at low $Q$ where $\alpha_{s}$ becomes large $\left(\alpha_{s} \approx 1\right.$ at $\left.Q \lesssim 1 \mathrm{GeV}\right)$. Thus, studying $\alpha_{s}$ in the strong QCD (sQCD) regime has been and remains an active field of research. Reaching the sQCD regime where pQCD fails is signaled by the unphysical divergence of $\alpha_{s}(Q)$ near $Q \approx \Lambda_{s}$ (the Landau pole), where $\Lambda_{s}$ is the scale driving the pQCD logarithmic evolution of $\alpha_{s}(Q)$. Owing to the fact that $Q \approx \Lambda_{s}$ signals the breakdown of pQCD -presumably due to the nonperturbative confinement effects$\Lambda_{s}$ is also understood as the momentum scale characterizing confinement. We

This material is based upon work supported by the U.S. Department of Energy, Office of Science, Office of Nuclear Physics under contract DE-AC05-06OR23177.

A. Deur

Thomas Jefferson National Accelerator Facility

Tel.: +757-269-7526

E-mail: deurpam@jlab.org 
discuss here works done in the low $Q$ regime, and reported in Refs. [1]-[6]. Ref. [7] provides a recent review of $\alpha_{s}$ in both small and large $Q$.

\section{The QCD coupling $\alpha_{s}$ in long distance regime}

Different definitions of $\alpha_{s}$ in the sQCD domain are possible and are in fact used [7]. In this document, the "effective charge" definition is employed [8]. It defines $\alpha_{s}$ from an observable's perturbative series truncated to its first order in $\alpha_{s}$, and is analogous to QED's coupling definition (Gell-Mann Low coupling [9]). As an example we apply below this prescription to the Bjorken sum rule [10], a fundamental relation for spin-dependent deep inelastic scattering. The pQCD approximant of the Bjorken sum rule is

$$
\begin{gathered}
\Gamma_{1}^{p-n}\left(Q^{2}\right) \equiv \int_{0}^{1}\left[g_{1}^{p}\left(x, Q^{2}\right)-g_{1}^{n}\left(x, Q^{2}\right)\right] d x= \\
\frac{g_{A}}{6}\left[1-\frac{\alpha_{\overline{M S}}\left(Q^{2}\right)}{\pi}-3.58\left(\frac{\alpha_{\overline{M S}}\left(Q^{2}\right)}{\pi}\right)^{2}-\cdots\right]+\sum_{i=2} \frac{\mu_{2 i}\left(Q^{2}\right)}{Q^{2 i}},
\end{gathered}
$$

where $g_{1}^{p, n}\left(x, Q^{2}\right)$ are the longitudinal spin structure functions for the proton and the neutron, $x$ the Bjorken scaling variable and $g_{A}$ the nucleon axial charge. The $\mu_{2 i}\left(Q^{2}\right)$ are nonperturbative higher-twist terms related to the confinement force [11]. They become important for $Q \lesssim 1 \mathrm{GeV}$. In Eq. (1), the $\alpha_{s}$ and the series coefficients are expressed in the $\overline{M S}$ renormalization scheme (RS). Using the effective charge definition, the Bjorken sum rule becomes

$$
\Gamma_{1}^{p-n}\left(Q^{2}\right) \equiv \int_{0}^{1}\left[g_{1}^{p}\left(x, Q^{2}\right)-g_{1}^{n}\left(x, Q^{2}\right)\right] d x \equiv \frac{g_{A}}{6}\left[1-\frac{\alpha_{g_{1}}\left(Q^{2}\right)}{\pi}\right],
$$

where the subscript $g_{1}$ for $\alpha_{g_{1}}\left(Q^{2}\right)$ indicates the observable chosen for the effective charge definition. This choice can be understood as equivalent to a RS choice [4]. With this prescription, the short distance pQCD effects (the terms of second and higher orders in $\alpha_{\overline{M S}}$ in Eq. (1)) and long distance confinement effects ( $\mu_{2 i}$ terms) are folded into $\alpha_{g_{1}}$. This is analogous to what transmutes the coupling constant in a classical lagrangian into a running effective coupling, i.e. when short distance quantum effects are folded into the coupling definition in the renormalization process [7]. The inclusion of the long distance confinement effects removes the Landau pole of $\alpha_{s}$ [7]. Thus, the effective charge definition is akin to the renormalization process, with the long distance effects regularizing $\alpha_{s}$.

There are several advantages offered by effective charges: they improve the convergence of the pQCD series, are extractable at any scale, are free of divergence and are RS-independent. This last characteristic arises because the first order coefficient of a pQCD series is RS-independent. However, the price to pay for these benefits is that an effective charge depends upon the process chosen for its definition: They are a priori different effective charges 
for different processes. However, QCD predictability is retained -at least in the perturbative domain- since effective charges are related by commensurate scale relations [12]. As already mentioned, this process-dependence is in fact equivalent to a particular choice of RS in the perturbative definition of $\alpha_{s}$.

Among the observables that can be used to define effective charges, the Bjorken sum $\Gamma_{1}^{p-n}$ is particularly interesting: the Bjorken sum rule at finite $Q^{2}$ has a relatively simple perturbative series, estimated up to $\alpha \frac{5}{M S}$ [13]. Furthermore, experimental data on $\Gamma_{1}^{p-n}$ exist at low, intermediate, and high $Q^{2}$ [14]. Finally, the rigorous Bjorken [10] and Gerasimov-Drell-Hearn (GDH) [15] sum rules dictate the behavior of $\Gamma_{1}^{p-n}$ in the unmeasured $Q^{2} \rightarrow 0$ and $Q^{2} \rightarrow \infty$ limits, respectively. These sum rules therefore supplement the data in these domains that are in practice unreachable by experiments. Consequently $\alpha_{g_{1}}$, the effective charge defined using $\Gamma_{1}^{p-n}$, is known at any $Q^{2}$. The experimental data supplemented by the sum rules are shown in Fig. 1. The data agree

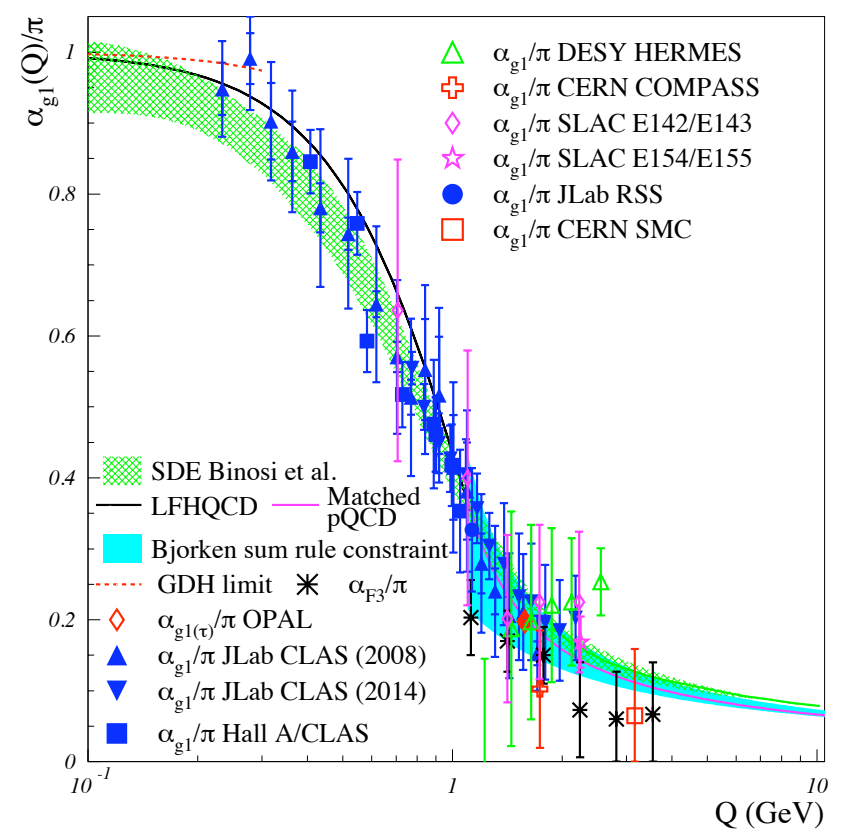

Fig. 1 The effective charge $\alpha_{g_{1}}(Q) / \pi$. The symbols indicate experimental data [1]. The dashed red line and solid blue band are the GDH [15] and Bjorken [10] sum rule predictions, respectively. The continuous black line is the LFHQCD computation [2], with its continuation to the pQCD domain $[3,5]$ shown by the continuous magenta line The green hatched band is the SDE calculation [16]. Comparisons with Lattice QCD calculations, older SDE results and various models are available in Ref. [7].

well with the process-independent Schwinger-Dyson Equation (SDE) calculation from Ref. [16] (see also the contribution of J. Rodriguez-Quintero to these proceedings [17]) and with the Light-Front Holographic QCD (LFHQCD) cal- 
culation, which will be discussed in the next Sections. Possible reasons for why the process-depend effective charge $\alpha_{g_{1}}$ agrees with these process-independent calculations are discussed in Ref. [7]. Finally, we note that Eq. (2) and that $\Gamma_{1}\left(Q^{2}\right) \underset{Q^{2} \rightarrow 0}{\longrightarrow} 0$ impose $\alpha_{g_{1}}\left(Q^{2}=0\right)=\pi$. $\Gamma_{1}$ vanishes because, as $Q^{2} \rightarrow 0$, all reactions of invariant mass $W$ are sent to $x \rightarrow 0$. Hence the only contribution to the $\Gamma_{1}$ integral is at $x=0$, i.e. infinite energy where cross-sections are zero. Thus, we will refer to the $\alpha_{g_{1}}(0)=\pi$ relation as a kinematic constraint

\section{The Light-Front Holographic QCD approximation}

Light-Front Holographic QCD (LFHQCD) [18] is an approximation to QCD based on light-front (LF) quantization [19]. This latter provides an exact and rigorous formulation of QCD, providing in particular a relativistic Schrödinger equation that describes hadrons as quark bound-states. All elements of the equation -including the confining potential term- can be in principle obtained from the QCD lagrangian; In practice however, we know how to compute it only in $(1+1)$ dimensions [20]. The overwhelming complexity of the analytic calculations in $(3+1)$ dimensions compels us to determine the potential with methods other than first-principle computations. A possibility is to use the correspondence between QCD on the LF and gravity in anti-de Sitter (AdS) space [21] based on the AdS/CFT (conformal field theory) duality [22]. The correspondence stems from the duality between a CFT in Minkowski spacetime and a corresponding group of isometries of a 5-dimensional AdS spacetime. Thanks to this correspondence, sQCD calculations become tractable in the chiral sector (quark masses set to zero) and if short-distance quantum fluctuations are neglected [18]. The projection of the 5-dimensional AdS calculations onto the AdS space boundary (a 4-dimensional spacetime identified with the physical Minkowski spacetime) provides a semiclassical approximation to sQCD that incorporates its fundamental aspects. In particular, it encodes by essence the conformal invariance of the classical QCD lagrangian, i.e. the fact that the lagrangian displays no explicit energy or distance scales in its expression.

The potential of the Schrödinger equation can be determined using the de Alfaro, Fubini, Furlan (dAFF) procedure [23], which allows the inclusion of a scale in a lagrangian without explicitly breaking the conformal symmetry of the resulting action. The demand of explicitly preserving conformal symmetry in effect restricts the potential to a unique form [24]; that of a harmonic oscillator on the LF, to which is added a spin-dependent term determined by the spin representations in AdS space. Only this form yields a massless pion in the chiral sector [25]. Furthermore, it explains the intriguing mass symmetry between baryons and mesons and predicts tetraquark spectroscopy [26,27]. Finally, a harmonic oscillator potential on the LF is equivalent in the usual instant-form front to the phenomenologically well-established linear potential for static quarks [28]. 
A single free parameter, the confinement scale $\kappa$, is used in LFHQCD. In fact, any theory or model describing QCD must have a least one free parameter since chiral QCD must be independent of conventional (human-chosen) units such as GeV. For pQCD, this single free parameter is $\Lambda_{s}$. For LFHQCD, it is $\kappa$. The relation between $\Lambda_{s}$ and $\kappa$ is analytically and numerically known [3] and its determination is a consequence of studying $\alpha_{s}$ at low $Q^{2}$. We will now show how it is determined in this regime using LFHQCD and how the relation between $\Lambda_{s}$ and $\kappa$ is obtained.

\section{LFHQCD computation of $\alpha_{s}\left(Q^{2}\right)$}

The measurement of $\alpha_{g_{1}}$ at low $Q^{2}$ and the kinematic constraint that $\alpha_{g_{1}}\left(Q^{2}=\right.$ $0)=\pi$ indicate that there, $\alpha_{g_{1}}\left(Q^{2}\right)$ is nearly constant, viz QCD is approximately conformal at low $Q^{2}$. (QCD is also nearly conformal at high $Q^{2}$ : it is the mildly violated Bjorken scaling). Such behavior of $\alpha_{g_{1}}\left(Q^{2}\right)$ at low $Q^{2}$ could also be deduced from the GDH sum rule [15] which predicts that:

$$
\frac{d \alpha_{g_{1}}\left(Q^{2}\right)}{d Q^{2}} \underset{Q^{2} \rightarrow 0}{\longrightarrow} \frac{3 \pi}{4 g_{A}}\left(\frac{\kappa_{p}^{2}}{M_{p}^{2}}-\frac{\kappa_{n}^{2}}{M_{n}^{2}}\right) .
$$

where $\kappa_{p}=1.79$ is the proton anomalous magnetic moment, $\kappa_{n}=-1.91$ the neutron one, and $M_{p, n}$ their respective masses. That $\left|\kappa_{n}\right| \approx \kappa_{p}$ implies that $d \alpha_{g_{1}} / d Q^{2} \underset{Q^{2} \rightarrow 0}{\longrightarrow} \approx 0$, and hence that QCD is nearly conformal at low $Q^{2}$. This fact allows us to apply LFHQCD to compute $\alpha_{g_{1}}\left(Q^{2}\right)[2]$.

The AdS action is similar to the Einstein-Hilbert action of General Relativity, except for being 5-dimensional:

$$
S_{A d S}=-\frac{1}{4} \int \sqrt{g} \frac{1}{a_{5}^{2}} F^{2} d^{5} x
$$

where $g=\operatorname{det}\left(g_{\mu \nu}\right) ; g_{\mu \nu}$ being the AdS space metric, $a_{5}$ is the coupling in AdS space and $F$ the gauge field. A momentum scale (e.g. $\kappa$ or $\Lambda_{s}$ ) characterizes QCD's confining potential, which thus breaks conformal symmetry. Hence the equivalent of the potential in Minkowski space is a distortion of its corresponding AdS space. As discussed in the previous section, there is a unique color confining potential, corresponding to a specific dilatation of $\mathrm{AdS}_{5}$ space, which retains the conformal symmetry of the action, as in the dAFF procedure [23]. This distortion takes the form of an exponential term $e^{\kappa^{2} z^{2}}$ factorizing the AdS interval. Thus, the action becomes

$$
S_{A d S}=-\frac{1}{4} \int \sqrt{g} \frac{1}{a_{5}^{2}} F^{2} e^{\kappa^{2} z^{2}} d^{5} x
$$

where $z$ is the fifth dimension of AdS space. $z^{2}$ gives the scale at which the hadron is probed, i.e. it corresponds to $1 / Q^{2}$. The universal LFHQCD scale factor $\kappa$ is obtained from either a hadron mass (e.g. $\kappa=M_{\rho} / 2$, with $M_{\rho}$ the 
$\rho$-meson mass) [18], a pion or nucleon form factor $[18,32]$ or $\Lambda_{s}[5]$. Its value is $\kappa=0.523 \pm 0.024 \mathrm{GeV}$ [33].

We explained in Section 2 that, just like in pQCD in which the short distance QCD effects (vacuum polarization) folded into the definition of the coupling constant $\alpha_{s}$ produce an effective running coupling $\alpha_{s}\left(Q^{2}\right)$, in SQCD, the long distance confinement forces are included into the effective charge definition. To follow this definition, the AdS space distortion factor equivalent to the QCD confinement potential is included in the coupling definition: $a_{5}^{e f f^{2}} \equiv a_{5}^{2} e^{-\kappa^{2} z^{2}}$. Transforming to the Minkowski momentum space yields

$$
\alpha_{s}^{L F H}\left(Q^{2}\right)=\alpha_{s}^{L F H}(0) e^{-\frac{Q^{2}}{4 \kappa^{2}}}
$$

where $\alpha_{s}^{L F H}(0)$ is undetermined. The kinematic constraint $\alpha_{g_{1}}(0)=\pi$ imposes $\alpha_{s}^{L F H}(0)=\pi$ for the $g_{1}$ scheme. The factor $\alpha_{s}^{L F H}(0)$ thus represents the observable-dependence of effective charges previously discussed, which is akin to the RS-dependence of $\alpha_{s}$ in pQCD.

The LFHQCD prediction, Eq. (6), agrees remarkably well with the low $Q^{2}$ data, see Fig. 1, while having no adjustable parameters, $\kappa$ and $c$ being imposed by hadron masses (or form factors) and kinematic constraint, respectively.

The expected domain of validity of the LFHQCD prediction is up to $Q^{2} \simeq 1$ $\mathrm{GeV}^{2}$. As mentioned in the previous Section, short distance quantum effects are not presently included in LFHQCD. Thus, LFHQCD is unsuited for high $Q^{2}$ phenomenology. Furthermore, in general AdS/CFT dualities demand the CFT coupling to be large, which is another reason why LFHQCD is not suited for large $Q^{2}$ calculations. However, $\alpha_{g_{1}}$ can be computed in this domain using pQCD. In fact, the applicability domains of pQCD and LFHQCD seem to overlap around $Q^{2} \simeq 1 \mathrm{GeV}^{2}$ [4]. This permits us to match the $\alpha_{g_{1}}$ calculation from LFHQCD (denoted $\alpha_{g_{1}}^{L F H}$ ) to that from pQCD (denoted $\alpha_{g_{1}}^{p Q C D}$ ). Specifically, we require that at a scale $Q_{0}$,

$$
\begin{array}{r}
\alpha_{g_{1}}^{L F H}\left(Q_{0}\right)=\alpha_{g_{1}}^{p Q C D}\left(Q_{0}\right) \text { and } \\
\left.\frac{d \alpha_{g_{1}}^{L F H}(Q)}{d Q}\right|_{Q=Q_{0}}=\left.\frac{d \alpha_{g_{1}}^{p Q C D}(Q)}{d Q}\right|_{Q=Q_{0} .} .
\end{array}
$$

$Q_{0}$ can be interpreted as the scale that sets the interface between the perturbative and nonperturbative domains [4]. It is thus where the DGLAP [29] and ERBL [30] evolutions begin, see e.g. Ref. [31] for an application of this concept. The existence of the domain overlap is validated a posteriori by the existence of a solution to Eqs (7). The solution provides both $Q_{0}$ and the relation between $\kappa$ and $\Lambda_{s}$ [3]. Since there is a direct relation between $\kappa$ and hadron masses [18], this in turn provides the determination of the hadron mass spectrum in terms of $\Lambda_{s}$. The relation between $M_{\rho}$ and $\Lambda_{\overline{M S}}$ is, at leading order [3]

$$
\Lambda_{\overline{M S}}=\frac{M_{\rho} e^{-a}}{\sqrt{a}},
$$


where $a=4\left[\sqrt{\ln ^{2}(2)+\beta_{0} / 4+1}-\ln (2)\right] / \beta_{0}$, with $\beta_{0}=11-2 n_{f} / 3$ the first coefficient of the $\beta$-series of QCD. Would QCD be exactly conformal, the $\beta$ function (and thus $\beta_{0}$ ) would be zero and $a \rightarrow \infty$. Eq. (8) then implies $\Lambda_{s} \rightarrow 0$, as expected in a conformal theory. For actual QCD with $n_{f}=3$ quark flavors, $a \simeq 0.55$ at $\mathrm{LO}$. At $\mathrm{N}^{3} \mathrm{LO}$ the relation must be solved numerically and is $\Lambda_{\overline{M S}}=0.440 M_{\rho}$.

The $\rho$ meson state is the solution of the LF Schrödinger equation with internal orbital angular momentum $L=0$ and radial excitation $n=0$. Higher mass mesons of the same family are solutions with $L>0$ or/and $n>0$. They can thus be obtained following the same method. The result at $\mathrm{N}^{3} \mathrm{LO}$ is shown in Fig. 2 together with the prediction for strange mesons obtained similarly. Baryonic masses can be obtained likewise, or using the mass symmetry between $L_{B}$ baryons and mesons with $L_{M}=L_{B}+1$ predicted by the superconformal algebraic structure in LFHQCD [26]. Hence, the method presented here provides an analytic determination of hadron spectrum with $\Lambda_{s}$ as the single input. The method can be reversed, using the known value of $\kappa$ to predict $\Lambda_{s}$. This yields $\Lambda_{\frac{n_{f}}{M S}}^{n^{2}}=0.339(19) \mathrm{GeV}$, in excellent agreement with the Particle Data Group average of 0.332(17) GeV [34].
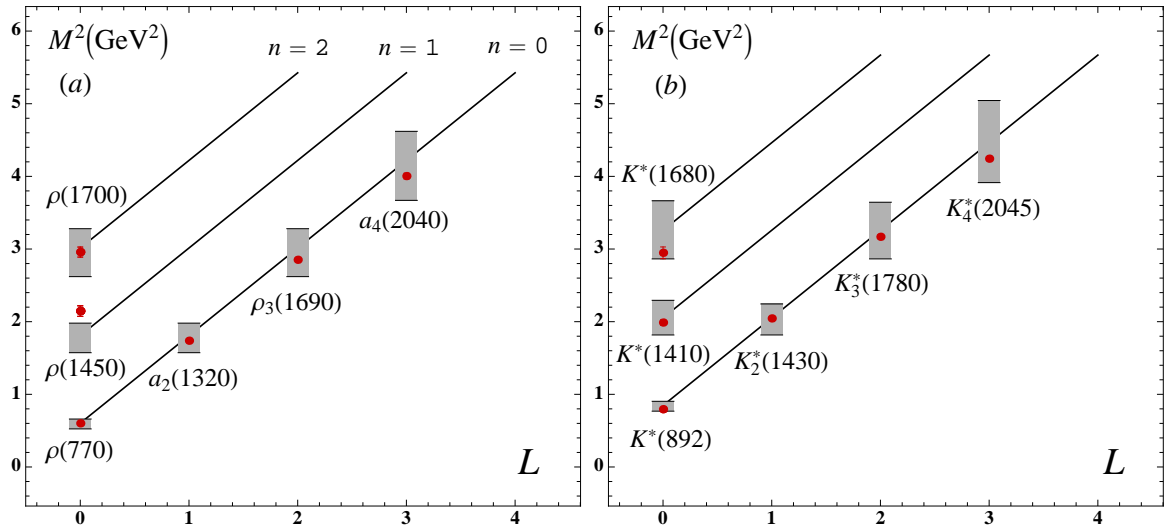

Fig. 2 The LFHQCD prediction of the mass spectrum for unflavored (left) and strange (right) light vector mesons. The gray rectangles indicate the uncertainty on the LFHQCD calculation. The red points are the experimental data.

\section{Summary and conclusion}

The coupling $\alpha_{s}\left(Q^{2}\right)$ is a central element of QCD. Vigorous research efforts aim to understand it in the strong QCD regime [7]. One such endeavor uses the Bjorken sum rule [10] to define an effective charge $\alpha_{g_{1}}\left(Q^{2}\right)$ [8]. Such choice is advantageous because data [14] and sum rules [10,15] allow us to obtain $\alpha_{g_{1}}\left(Q^{2}\right)$ over all $Q^{2}[1]$. Both the experimental data and the sum rules independently indicate that QCD is nearly conformal at low $Q^{2}$. This makes 
possible to compute strong QCD using the AdS/CFT correspondence [22], in particular in its LFHQCD incarnation [21]. This provides a semiclassical analytic approach to strong QCD that is fully determined and can be used to solve QCD on the light-front [18]. The potential arising in the (rigorous) light-front bound-state equation is imposed by explicitly respecting QCD's conformal symmetry [23]. Only a single free parameter, $\kappa$, is needed, which is in fact the minimal amount of parameters necessary for any theory or model aiming to describe the strong interaction. The strong coupling $\alpha_{g_{1}}\left(Q^{2}\right)$ obtained with LFHQCD [2] has no adjustable parameter and is in remarkable agreement with the experimental data [1] and with calculations form different approaches to strong QCD [7], including the recent Schwinger-Dyson Equation calculation of a process independent coupling $[16,17]$. The fact that the validity domains of LFHQCD and pQCD overlap around $Q^{2}=1 \mathrm{GeV}^{2}$ enables us to obtain both the scale $Q_{0}$ that sets the interface between the nonperturbative and perturbative domains $[4,31]$ and an analytic determination of the hadron mass spectrum from the fundamental QCD parameter $\Lambda_{s}$ [3]. Conversely, it permits a high-accuracy determination of $\Lambda_{s}[4,5]$ that agrees well with the world average [34].

Being able to analytically compute the hadron mass spectrum has been a long thought goal and would signal that we finally managed to analytically solved QCD. While LFHQCD is not QCD but a semi-classical approximation of it, its premises are based on the rigorous LF approach to quantum field theory and the formal relation between the isometry group of a 5-dimensional AdS space and the conformal invariance of the dual theory in the 4-dimensional physical space. The foundations of LFHQCD are thus solid and the analytic computation of the hadron spectrum is an encouraging progress toward the ultimate goal of analytically solving QCD.

Acknowledgements This work was done in collaboration with S. J. Brodsky, V. Burkert, J-P Chen, G. de Téramond and W. Korsch. We thank S. J. Brodsky and G. de Téramond for reading the manuscript and providing useful comments.

\section{References}

1. A. Deur, V. Burkert, J. P. Chen and W. Korsch, "Experimental determination of the effective strong coupling constant," Phys. Lett. B 650, 244 (2007); [hep-ph/0509113]; "Determination of the effective strong coupling constant $\alpha_{s, g_{1}}\left(Q^{2}\right)$ from CLAS spin structure function data," Phys. Lett. B 665, 349 (2008) [arXiv:0803.4119 [hep-ph]].

2. S. J. Brodsky, G. F. de Téramond and A. Deur, Nonperturbative QCD Coupling and its $\beta$-function from Light-Front Holography, Phys. Rev. D 81, 096010 (2010) [arXiv:1002.3948 [hep-ph]]

3. A. Deur, S. J. Brodsky and G. F. de Téramond, Connecting the hadron mass scale to the fundamental mass scale of quantum chromodynamics, Phys. Lett. B 750, 528 (2015) [arXiv:1409.5488 [hep-ph]]

4. A. Deur, S. J. Brodsky and G. F. de Téramond, On the interface between perturbative and nonperturbative QCD, Phys. Lett. B 757, 275 (2016) [arXiv:1601.06568 [hep-ph]];

5. A. Deur, S. J. Brodsky and G. F. de Téramond, "Determination of $\Lambda_{\bar{M} S}$ at five loops from holographic QCD," J. Phys. G 44, no. 10, 105005 (2017) arXiv:1608.04933 [hep-ph]. 
6. A. Deur, J. M. Shen, X. G. Wu, S. J. Brodsky and G. F. de Téramond, "Implications of the Principle of Maximum Conformality for the QCD Strong Coupling," Phys. Lett. B 773, 98 (2017) [arXiv:1705.02384 [hep-ph]].

7. A. Deur, S. J. Brodsky and G. F. de Téramond, "The QCD Running Coupling," Prog. Part. Nucl. Phys. 90, 1 (2016) [arXiv:1604.08082 [hep-ph]].

8. G. Grunberg, "Renormalization Group Improved Perturbative QCD," Phys. Lett. 95B, 70 (1980) Erratum: [Phys. Lett. 110B, 501 (1982)]; G. Grunberg, "Renormalization Scheme Independent QCD and QED: The Method of effective charges," Phys. Rev. D 29, 2315 (1984); G. Grunberg, "On Some Ambiguities in the Method of effective charges," Phys. Rev. D 40, 680 (1989).

9. M. Gell-Mann and F. E. Low, Quantum electrodynamics at small distances, Phys. Rev. 95, 1300 (1954).

10. J. D. Bjorken, Applications of the Chiral $U(6) \times U(6)$ Algebra of Current Densities, Phys. Rev. 148, 1467 (1966); Inelastic Scattering of Polarized Leptons from Polarized Nucleons, Phys. Rev. D 1, 1376 (1970).

11. M. Burkardt, "Transverse force on quarks in deep-inelastic scattering," Phys. Rev. D 88, 114502 (2013) [arXiv:0810.3589 [hep-ph]]. M. Abdallah and M. Burkardt, "Transverse Force on Transversely Polarized Quarks in Longitudinally Polarized Nucleons," Phys. Rev. D 94, no. 9, 094040 (2016) [arXiv:1610.01166 [hep-ph]].

12. S. J. Brodsky and H. J. Lu, Commensurate scale relations in quantum chromodynamics, Phys. Rev. D 51, 3652 (1995) [hep-ph/9405218];

13. A. L. Kataev, The Ellis-Jaffe sum rule: The estimates of the next to next-to-leading order QCD corrections, Phys. Rev. D 50, 5469 (1994) [hep-ph/9408248]; A. L. Kataev, "Deep inelastic sum rules at the boundaries between perturbative and nonperturbative QCD," Mod. Phys. Lett. A 20, 2007 (2005) [hep-ph/0505230]; P. A. Baikov, K. G. Chetyrkin and J. H. Kuhn, Adler function, Bjorken sum rule, and the Crewther relation to order $\alpha_{s}^{4}$ in a general gauge theory, Phys. Rev. Lett. 104, 132004 (2010) [arXiv:1001.3606 [hep-ph]].

14. B. Adeva et al. [Spin Muon Collaboration], "Measurement of the spin dependent structure function $\mathrm{g} 1(\mathrm{x})$ of the deuteron," Phys. Lett. B 302, 533 (1993); "The Spin dependent structure function $\mathrm{g}(1)(\mathrm{x})$ of the proton from polarized deep inelastic muon scattering," Phys. Lett. B 412, 414 (1997); D. Adams et al. [Spin Muon (SMC) Collaboration], "Measurement of the spin dependent structure function $g_{1}(x)$ of the proton," Phys. Lett. B 329 399 (1994) Erratum: [Phys. Lett. B 339, 332 (1994)] [hep-ph/9404270]; "A New measurement of the spin dependent structure function $\mathrm{g} 1(\mathrm{x})$ of the deuteron," Phys. Lett. B 357, 248 (1995); "The Spin dependent structure function $\mathrm{g} 1(\mathrm{x})$ of the deuteron from polarized deep inelastic muon scattering," Phys. Lett. B 396, 338 (1997); "Spin structure of the proton from polarized inclusive deep inelastic muon - proton scattering," Phys. Rev. D 56, 5330 (1997) [hep-ex/9702005]; P. L. Anthony et al. [E142 Collaboration], "Deep inelastic scattering of polarized electrons by polarized He-3 and the study of the neutron spin structure," Phys. Rev. D 54, 6620 (1996) [hep-ex/9610007]; K. Abe et al. [E143 Collaboration], "Precision measurement of the proton spin structure function g1(p)," Phys. Rev. Lett. 74, 346 (1995); "Precision measurement of the deuteron spin structure function g1(d)," Phys. Rev. Lett. 75, 25 (1995); "Measurements of the $Q^{2}$ dependence of the proton and deuteron spin structure functions g1(p) and g1(d)," Phys. Lett. B 364, 61 (1995) [hep-ex/9511015]; "Measurements of the proton and deuteron spin structure function $\mathrm{g} 1$ in the resonance region," Phys. Rev. Lett. 78, 815 (1997) [hep-ex/9701004]; "Measurements of the proton and deuteron spin structure functions g(1) and g(2)," Phys. Rev. D 58, 112003 (1998) [hepph/9802357]; K. Abe et al. [E154 Collaboration], "Precision determination of the neutron spin structure function g1(n)," Phys. Rev. Lett. 79, 26 (1997) [hep-ex/9705012]; "Next-toleading order QCD analysis of polarized deep inelastic scattering data," Phys. Lett. B 405, 180 (1997) [hep-ph/9705344]; P. L. Anthony et al. [E155 Collaboration], "Measurement of the deuteron spin structure function $\mathrm{g} 1(\mathrm{~d})(\mathrm{x})$ for $1-(\mathrm{GeV} / \mathrm{c})^{2}<Q^{2}<40-(\mathrm{GeV} / \mathrm{c})^{2}$," Phys. Lett. B 463, 339 (1999) [hep-ex/9904002]; "Measurements of the $Q^{2}$ dependence of the proton and neutron spin structure functions $\mathrm{g}(1)^{p}$ and $\mathrm{g}(1)^{n}$," Phys. Lett. B 493, 19 (2000) [hep-ph/0007248]. A. Airapetian et al. [HERMES Collaboration], "The $Q^{2}$ dependence of the generalized Gerasimov-Drell-Hearn integral for the proton," Phys. Lett. B 494, 1 (2000) [hep-ex/0008037]; "The $Q^{2}$ dependence of the generalized GerasimovDrell-Hearn integral for the deuteron, proton and neutron," Eur. Phys. J. C 26, 527 
(2003) [hep-ex/0210047]; K. Ackerstaff et al. [HERMES Collaboration], "Determination of the deep inelastic contribution to the generalized Gerasimov-Drell-Hearn integral for the proton and neutron," Phys. Lett. B 444, 531 (1998) [hep-ex/9809015]; A. Deur et al., "Experimental determination of the evolution of the Bjorken integral at low $Q^{2}$," Phys. Rev. Lett. 93, 212001 (2004) [hep-ex/0407007]; "High precision determination of the $Q^{2}$ evolution of the Bjorken Sum," Phys. Rev. D 90, no. 1, 012009 (2014) [arXiv:1405.7854 [nucl-ex]]; A. Deur et al., "Experimental study of isovector spin sum rules," Phys. Rev. D 78, 032001 (2008) [arXiv:0802.3198 [nucl-ex]]; F. R. Wesselmann et al. [RSS Collaboration], "Proton spin structure in the resonance region," Phys. Rev. Lett. 98, 132003 (2007) [nucl-ex/0608003]; K. Slifer et al. [Resonance Spin Structure Collaboration], "Probing Quark-Gluon Interactions with Transverse Polarized Scattering," Phys. Rev. Lett. 105, 101601 (2010) [arXiv:0812.0031 [nucl-ex]]; M. G. Alekseev et al. [COMPASS Collaboration], "The Spin-dependent Structure Function of the Proton $g_{1}^{p}$ and a Test of the Bjorken Sum Rule," Phys. Lett. B 690, 466 (2010) [arXiv:1001.4654 [hep-ex]].

15. S. B. Gerasimov, A Sum rule for magnetic moments and the damping of the nucleon magnetic moment in nuclei, Sov. J. Nucl. Phys. 2, 430 (1966) [Yad. Fiz. 2, 598 (1965)]; S. D. Drell and A. C. Hearn, Exact Sum Rule for Nucleon Magnetic Moments, Phys. Rev Lett. 16, 908 (1966); M. Hosoda and K. Yamamoto Sum Rule for the Magnetic Moment of the Dirac Particle, Prog. Theor. Phys. 36 (2), 425 (1966).

16. D. Binosi, C. Mezrag, J. Papavassiliou, C. D. Roberts and J. Rodriguez-Quintero, "Process-independent strong running coupling," Phys. Rev. D 96, no. 5, 054026 (2017) [arXiv:1612.04835 [nucl-th]].

17. J. Rodrguez-Quintero, D. Binosi, C. Mezrag, J. Papavassiliou and C. D. Roberts, "Process-independent effective coupling. From QCD Green's functions to phenomenology," arXiv:1801.10164 [nucl-th].

18. S. J. Brodsky, G. F. de Téramond, H. G. Dosch and J. Erlich, "Light-Front Holographic QCD and Emerging Confinement," Phys. Rept. 584, 1 (2015) [arXiv:1407.8131 [hep-ph]].

19. S. J. Brodsky, H. C. Pauli and S. S. Pinsky, "Quantum chromodynamics and other field theories on the light-cone," Phys. Rept. 301, 299 (1998) [hep-ph/9705477].

20. K. Hornbostel, S. J. Brodsky and H. C. Pauli, "light-cone Quantized QCD in (1+1)Dimensions," Phys. Rev. D 41, 3814 (1990).

21. S. J. Brodsky and G. F. de Téramond, "Hadronic spectra and light-front wavefunctions in holographic QCD," Phys. Rev. Lett. 96, 201601 (2006) [hep-ph/0602252]; G. F. de Teramond and S. J. Brodsky, "Light-Front Holography: A First Approximation to QCD," Phys. Rev. Lett. 102, 081601 (2009) [arXiv:0809.4899 [hep-ph]].

22. J. M. Maldacena, "The Large N limit of superconformal field theories and supergravity," Int. J. Theor. Phys. 38, 1113 (1999) [Adv. Theor. Math. Phys. 2, 231 (1998)] [hepth/9711200];

23. V. de Alfaro, S. Fubini and G. Furlan, Conformal invariance in quantum mechanics, Nuovo Cim. A 34, 569 (1976). S. Fubini and E. Rabinovici, Superconformal quantum mechanics, Nucl. Phys. B 245, 17 (1984). V. P. Akulov and A. I. Pashnev, Quantum superconformal model in $(1,2)$ space, Theor. Math. Phys. 56, 862 (1983) [Teor. Mat. Fiz. 56, $344(1983)]$.

24. S. J. Brodsky, G. F. De Téramond and H. G. Dosch, "Threefold Complementary Approach to Holographic QCD," Phys. Lett. B 729, 3 (2014) [arXiv:1302.4105 [hep-th]].

25. H. G. Dosch, G. F. de Téramond and S. J. Brodsky, "Superconformal Baryon-Meson Symmetry and Light-Front Holographic QCD," Phys. Rev. D 91, no. 8, 085016 (2015) [arXiv:1501.00959 [hep-th]].

26. G. F. de Téramond, H. G. Dosch and S. J. Brodsky, "Baryon Spectrum from Superconformal Quantum Mechanics and its Light-Front Holographic Embedding," Phys. Rev. D 91, no. 4, 045040 (2015) [arXiv:1411.5243 [hep-ph]].

27. S. J. Brodsky, G. F. de Téramond, H. G. Dosch and C. Lorc, "Meson/Baryon/Tetraquark Supersymmetry from Superconformal Algebra and Light-Front Holography," Int. J. Mod. Phys. A 31, no. 19, 1630029 (2016) [arXiv:1606.04638 [hep$\mathrm{ph}]$.

28. A. P. Trawinski, S. D. Glazek, S. J. Brodsky, G. F. de Téramond and H. G. Dosch, "Effective confining potentials for QCD," Phys. Rev. D 90, no. 7, 074017 (2014) [arXiv:1403.5651 [hep-ph]]. 
29. V. N. Gribov and L. N. Lipatov, Deep inelastic e p scattering in perturbation theory, Sov. J. Nucl. Phys. 15, 438 (1972) [Yad. Fiz. 15, 781 (1972)]; G. Altarelli and G. Parisi, Asymptotic freedom in parton language, Nucl. Phys. B 126, 298 (1977); Y. L. Dokshitzer, Calculation of the structure functions for deep inelastic scattering and $e^{+} e^{-}$annihilation by perturbation theory in quantum chromodynamics, Sov. Phys. JETP 46, 641 (1977) [Zh. Eksp. Teor. Fiz. 73, 1216 (1977)].

30. G. P. Lepage and S. J. Brodsky, "Exclusive Processes in Quantum Chromodynamics: Evolution Equations for Hadronic Wave Functions and the Form-Factors of Mesons," Phys. Lett. 87B, 359 (1979). A. V. Efremov and A. V. Radyushkin, "Factorization and Asymptotical Behavior of Pion Form-Factor in QCD," Phys. Lett. 94B, 245 (1980).

31. G. F. de Téramond et al. [HLFHS Collaboration], "Universality of Generalized Parton Distributions in Light-Front Holographic QCD," arXiv:1801.09154 [hep-ph].

32. R. S. Sufian, G. F. de Téramond, S. J. Brodsky, A. Deur and H. G. Dosch, "Analysis of nucleon electromagnetic form factors from light-front holographic QCD : The spacelike region," Phys. Rev. D 95, no. 1, 014011 (2017) [arXiv:1609.06688 [hep-ph]].

33. S. J. Brodsky, G. F. de Téramond, H. G. Dosch and C. Lorcé "Universal Effective Hadron Dynamics from Superconformal Algebra," Phys. Lett. B 759, 171 (2016) [arXiv:1604.06746 [hep-ph]].

34. C. Patrignani et al. [Particle Data Group Collaboration], "Review of Particle Physics," Chin. Phys. C 40, no. 10, 100001 (2016) and 2017 update. 